\title{
Branched Chain Amino Acids Improve Complex Maze Learning in Rat Offspring Prenatally Exposed to Hyperphenylalaninemia: Implications for Maternal Phenylketonuria
}

\author{
CHARLES V. VORHEES AND HELEN K. BERRY \\ Institute for Developmental Research, Children's Hospital Research Foundation; and Department of Pediatrics, \\ University of Cincinnati, Cincinnati, $\mathrm{OH}$
}

\begin{abstract}
Maternal phenylketonuria results in a high incidence of children born who are mentally retarded. It has been suggested that blood-brain-barrier transport of phenylalanine may be reduced by competitive inhibition of transporter uptake by supplemental administration of other large neutral amino acids. We hypothesized that large neutral amino acids might also be effective at improving the outcome of fetuses exposed to hyperphenylalaninemia in utero. If correct, sparing of embryonic CNS development might be possible. Pregnant rats were given a hyperphenylalaninemic diet alone or the same diet supplemented with a combination of valine, isoleucine, and leucine. As adults, the progeny exposed in utero to hyperphenylalaninemia showed characteristic learning deficits in a complex maze, while those exposed in utero to hyperphenylalaninemia combined with valine, isoleucine, and leucine showed no deficits in maze acquisition. The valine, isoleucine, and leucine supplement may show promise as a treatment for intrauterinely acquired mental deficiency associated with maternal phenylketonuria. (Pediatr Res 25:568-572, 1989)
\end{abstract}

\section{Abbreviations}

PKU, phenylketonuria

LNAA, large neutral amino acid

PCPA, D,L-p-chlorophenylalanine

PFC, pair-fed control

VIL, valine, isoleucine, and leucine

PKU, an inherited disorder of phenylalanine metabolism, has been treated during the last $30 \mathrm{y}$ through a combination of phenylalanine-free formulas and dietary restriction of phenylalanine-containing foods (1). The result is an increasing number of nonmentally retarded, noninstitutionalized PKU adults starting families. Unfortunately, maternal PKU causes a high incidence of mental retardation and congenital defects in infants due to transplacental hyperphenylalaninemia (2-4). Relying upon evidence developed concerning blood brain barrier transport of amino acids, we sought to reduce the embryotoxic effects of hyperphenylalaninemia using the branched-chain amino acids in a murine model of PKU.

Received October 17, 1988; accepted February 23, 1989

Correspondence and reprints to Charles V. Vorhees, Ph.D., Institute for Developmental Research, Children's Hospital Research Foundation, Cincinnati, OH 45229-2899.

Supported by USPHS Grant HD 19090 from NIH.
A review of worldwide cases (2) found that $95 \%$ of pregnant PKU women with serum phenylalanine levels $\geq 20 \mathrm{mg} / \mathrm{dL}$ delivered at least one child who was mentally deficient. Other findings were microcephaly (90\%), growth retardation (56\%), and congenital heart defects $(40 \%)$. Reinstatement of a phenylalaninerestricted diet before or after recognition of pregnancy has produced mixed, but generally unsatisfactory, results (2-7).

This has led to the search for new approaches. Phenylalanine and other LNAA share a common transport carrier for selective CNS uptake (8-12). It has been established that increasing the concentration of one or more LNAA competitively inhibits the transport into brain of other amino acids in this group. This approach has been suggested as a means of reducing the influx of phenylalanine in PKU (13-18). Early experiments to assess this have shown promise $(13-15,17-22)$, but in no case was evidence provided that such approaches could produce lasting cognitive improvement. Given that mental retardation is the most prevalent untoward effect of maternal PKU, we sought to test the efficacy of the branched-chain LNAA to improve embryo-fetal development in pregnant hyperphenylalaninemic rats to determine if sparing of CNS development in the progeny was possible.

\section{MATERIALS AND METHODS}

Nulliparous female Sprague-Dawley CD (VAF/Plus) rats (Charles River Breeding Laboratories, Portage, MI) were bred and on $\mathrm{d} 4$ (sperm plug $=\mathrm{d} 0$ ) were randomly assigned to one of three treatment groups on a wt matched basis. Experimental diets were provided on gestation $\mathrm{d} 5-15$. The PKU group received diet containing $2.5 \%$ added L-phenylalanine (phe) and $0.12 \%$ PCPA by wt (23-25) ad libitum. PCPA is a phenylalanine hydroxylase inhibitor (26-27). The PKU-VIL group received the phe+PCPA diet ad libitum with VIL added at concentrations of $1.0 \% \mathrm{~L}$-valine, $1.0 \% \mathrm{~L}$-isoleucine, and $1.33 \%$ L-leucine by wt (18-21). The PFC group received the base diet in the amount consumed by its matched pair in the PKU group. Neither phe alone nor PCPA alone induces long-term effects on water maze performance or on any other measure of behavioral performance characteristic of PKU animal models using similar treatment regimens $(23,24,28-30)$. On $d 11$ and 15 of gestation, every other dam in each group was anesthetized with methoxyflurane and a blood sample drawn from the tail for determination of serum amino acids. Number of litters/group were: $\mathrm{PKU}=10$; PKU-VIL $=12 ;$ PFC $=12$

At birth, litters were adjusted to eight (four males and four females) using a random selection method. Offspring were weaned and housed in same-sexed pairs on postnatal d 28 . Offspring were assessed on four measures of neurobehavioral 
and cognitive functioning by personnel blind to treatment group assignment.

On d 50-52 all offspring were assessed for $15 \mathrm{~min} / \mathrm{d}$ in an automated open-field as a test of locomotor activity under lowlevel illumination during the diurnal phase after a 10 min dark adaptation period. Each rat was tested individually and at the same time each day. The fields were circular and had diameters of $45.7 \mathrm{~cm}$. Males and females were tested in separate fields, which were cleaned between subjects. There were four LEDphototransister pairs aligned in the $x$-axis and 4 in the $y$-axis of each field (San Diego Instruments, San Diego, CA).

On $\mathrm{d} 55$, half of the male and female offspring from each litter received four trials in a $150-\mathrm{cm}$ straight water channel to acclimate them to a swimming task, as a measure of performance capacity, and as a measure of their motivation to escape from water. Time to swim the channel and reach an escape ladder were analyzed by ANOVA for sum of trials, last trial, and fastest trial. Data for rats within each litter were averaged together and litter, stratified for sex, was the unit of statistical analysis (31). Water temperature was $22 \pm 1^{\circ} \mathrm{C}$.

Rats tested in the straight channel were next evaluated for learning in a complex water maze. The maze chosen was an expanded version of the Biel maze (32). Path A consisted of the sum of errors committed on six trials (two trials/d) given on $d$ $56-58$ on the initial maze problem (acquisition). Path B consisted of the sum of errors committed on six additional trials (two trials/d) administered on d 62-64 with the start and goal areas reversed (reversal). Two types of errors were recorded, cul-de-sac errors, which were whole body entries into any blind alley, and excursion errors, which were whole body entries into any channel of the maze other than the path leading to the goal. Elapsed maze times were also recorded (s). Four offspring (two males and two females) were tested/litter. Rats failing to reach the goal within 6 min were assisted to the goal by placing barriers in the maze behind them thereby preventing re-entry into previously chosen channels. Errors and maze times were analyzed by fixedeffect factorial ANOVA with litter, stratified for sex, as the unit of analysis (31).

On d 65 two males and two females/litter were tested for modified startle responses. Rats were tested in a San Diego Instruments SR apparatus. Rats were placed in a sound-attenuated chamber and acclimated for $5 \mathrm{~min}$ before their first trial. Each rat was given 72 trials. The first 36 trials used a white noise auditory prepulse stimulus of $90 \mathrm{~dB}(\mathrm{~A})$ and an auditory startleeliciting stimulus of $115 \mathrm{~dB}(\mathrm{~A})$ presented against a continuous $70 \mathrm{~dB}(\mathrm{~A})$ background noise. All signals were of mixed frequency with a predominant band at $4 \mathrm{kHz}$. The second 36 trials used the same auditory prepulse followed by a tactile startle-eliciting stimulus ( 12 psi air-puff). The interstimulus interval (gap) ranged from $0-4000 \mathrm{~ms}$ and were presented in a Latin square design with an intertrial interval of $30 \mathrm{~s}$. The first trial was always a noprepulse trial. Data for all trials with the same interstimulus interval were averaged together. Interstimulus gaps were measured from prepulse signal onset to startle stimulus onset. Signal duration was $20 \mathrm{~ms}$ and the response window was $100 \mathrm{~ms}$ from startle signal onset. Dependent measures were $\mathrm{V}_{\max }=$ maximum response (voltage change) occurring during the response window, $\mathrm{V}_{\text {mean }}=$ mean voltage change during the recording window taken each $\mathrm{ms}$, and $\mathrm{T}_{\max }=$ response latency (ms) from signal onset to $\mathrm{V}_{\max }$.

Serum amino acids were analyzed on a Beckman model 119B amino acid analyzer (Beckman Instruments Inc., Palo Alto, CA) (33-34). Offspring data were analyzed using the litter mean as the unit of statistical analysis (31). Statistical inference was based on general linear model ANOVA (factorial and mixed models, as appropriate), with follow-up analyses using simple-effect ANOVA and Duncan a posteriori multiple-group comparisons. Anesthesia/blood sampling history was a factor in a series of initial analyses. These uniformly demonstrated that there were no effects of this procedure on any dependent measure.

\section{RESULTS}

Serum amino acid patterns of dams in the PKU and PKUVIL groups exhibited several differences (Table 1). The serum concentrations of phenylalanine were in the phenylketonuric range at both time points and were greater on $d 15$ than on $\mathrm{d} 11$ in both groups. No differences in serum phenylalanine between the PKU and PKU-VIL groups were obtained confirming that VIL did not alter maternal phenylalanine or PCPA absorption or metabolism. As expected, the PKU-VIL group showed substantial increases in serum VIL. Minor differences were also noted between groups in serum threonine, serine, and glycine.

No differences were found in maternal food consumption throughout gestation. No differences in maternal body wt were observed from d $0-14$ of gestation. A minor effect on maternal wt appeared on d 15 and 20 (PKU and PKU-VIL groups compared to PFC, $p<0.10$ ). On the $\mathrm{d}$ of birth, but not thereafter, there was a group effect on maternal wt $(p<0.01)$, such that the

Table 1. Serum amino acids in hyperphenylalaninemic pregnant rat dams with and without branched chain dietary supplementation*

\begin{tabular}{|c|c|c|c|c|}
\hline \multirow{2}{*}{$\begin{array}{l}\text { Amino } \\
\text { Acid }\end{array}$} & \multicolumn{2}{|c|}{$\begin{array}{l}\text { Gestation d } 11(\mu \mathrm{mol} / \\
\text { liter })\end{array}$} & \multicolumn{2}{|c|}{ Gestation d 15 ( $\mu \mathrm{mol} /$ liter $)$} \\
\hline & PKU & PKU-VIL & PKU & PKU-VIL \\
\hline Tau & $399 \pm 53$ & $297 \pm 22$ & $399 \pm 51$ & $313 \pm 55$ \\
\hline Asp & $21 \pm 6$ & $13 \pm 2$ & $19 \pm 3$ & $12 \pm 3$ \\
\hline Thr & $319 \pm 25$ & $249 \pm 20 \dagger$ & $293 \pm 11$ & $259 \pm 10 \dagger$ \\
\hline Ser & $318 \pm 26$ & $241 \pm 15 \dagger$ & $263 \pm 24$ & $231 \pm 12 \dagger$ \\
\hline Asn & $65 \pm 9$ & $54 \pm 5$ & $62 \pm 6$ & $54 \pm 5$ \\
\hline Glu & $74 \pm 15$ & $51 \pm 7$ & $84 \pm 12$ & $66 \pm 10$ \\
\hline Gln & $556 \pm 29$ & $461 \pm 38$ & $473 \pm 26$ & $441 \pm 30$ \\
\hline Pro & $184 \pm 21$ & $166 \pm 16$ & $199 \pm 12$ & $206 \pm 16$ \\
\hline Gly & $254 \pm 21$ & $209 \pm 9 \dagger$ & $236 \pm 26$ & $190 \pm 15 \dagger$ \\
\hline Ala & $562 \pm 61$ & $497 \pm 36$ & $488 \pm 28$ & $525 \pm 14$ \\
\hline Cit & $79 \pm 3$ & $73 \pm 9$ & $43 \pm 6$ & $81 \pm 10$ \\
\hline Val & $192 \pm 14$ & $321 \pm 57 \ddagger$ & $204 \pm 10$ & $358 \pm 36 \ddagger$ \\
\hline Cys & $14 \pm 4$ & $27 \pm 5$ & $20 \pm 5$ & $22 \pm 9$ \\
\hline Met & $64 \pm 3$ & $55 \pm 3$ & $58 \pm 2$ & $52 \pm 8$ \\
\hline Ile & $87 \pm 5$ & $130 \pm 26 t$ & $86 \pm 3$ & $151 \pm 13 \neq$ \\
\hline Leu & $143 \pm 10$ & $205 \pm 38 \ddagger$ & $141 \pm 7$ & $239 \pm 20 \ddagger$ \\
\hline Tyr & $148 \pm 7$ & $128 \pm 9$ & $148 \pm 15$ & $152 \pm 12$ \\
\hline Phe & $1693 \pm 447$ & $2025 \pm 429$ & $2635 \pm 429 \S$ & $2920 \pm 426 \S$ \\
\hline PCPA & $365 \pm 32$ & $400 \pm 29 \dagger$ & $466 \pm 11$ & $555 \pm 29 \dagger$ \\
\hline Phe $m g / d L$ & $28 \pm 7$ & $33 \pm 7$ & $44 \pm 7$ & $48 \pm 7$ \\
\hline $\begin{array}{l}\text { Dams bled } \\
\text { in each } \\
\text { group } \\
(n)\end{array}$ & 7 & 7 & 7 & 7 \\
\hline
\end{tabular}

* Pregnant rats were given diets containing either $2.5 \%$ L-phenylalanine $+0.12 \% \mathrm{D}, \mathrm{L}-p$-chlorophenylalanine (PKU group) or the same amounts of phenylalanine + PCPA plus VIL $(1.0 \%$ L-valine, $1.0 \%$ isoleucine, and $1.33 \%$ L-leucine (PKU-VIL group) by wt) on d 5-15 of gestation. One-half of the dams in each group (including PFC) were anesthetized with methoxyflurane on $\mathrm{d} 11$ and 15 , and a blood sample was drawn from the tail. Samples were centrifuged, and serum was removed and frozen for later amino acid analysis. Amino acids were analyzed on a Beckman model 119B amino acid analyzer, including separation of PCPA. Amino acid concentrations were analyzed using treatment group $\times$ gestational age ANOVA. All maternal and offspring data were analyzed with anesthesia/blood sampling history as a factor in ANOVA for all dependent variables. These analyses showed no main effects of sampling history and no interactions of sampling history with treatment group on any dependent variable.

$\dagger p<0.05$ compared to PKU group.

$\ddagger p<0.01$ compared to PKU group.

$\S p<0.01$ increase compared to $\mathrm{d} 11$ levels. 
PKU dams weighed less than the PKU-VIL $(7.6 \% ; p<0.05)$ or PFC dams $(12.4 \% ; p<0.05)$. There were no differences in the number of delivering dams, litter size, or offspring sex ratio at birth among groups. There were no significant group differences in offspring body wt at any age. Offspring mortality was increased before weaning in the PKU group (8.8\%) and PKU-VIL group $(8.5 \%)$ compared to PFC $(2.1 \%$, both comparisons $p<0.05)$.

No differences in basic swimming performance were obtained in straight channel trials (Fig. 1). This indicated that the groups did not differ in swimming ability or motivation. In contrast, large differences occurred in maze performance (Fig. 2). Using two methods of scoring errors, the PKU offspring committed more $c u l-d e-s a c$ and excursion errors than PFC offspring on both phases of maze testing and required more time in the maze. Rats in the PKU-VIL group committed fewer errors than the PKU group and did not differ from PFCs on path $\mathrm{A}$. On path $\mathrm{B}$, the PKU-VIL group committed fewer errors than the PKU group and more errors than the PFC group; however, they did not commit significantly more errors than PFC or significantly fewer than PKU.

In the open-field test, there were group $\times \mathrm{d}$ and group $\times$ testinterval interactions for peripheral activity $(p<0.05$ and $p<$ 0.01 , respectively), but no effects on central section activity. Further analyses showed only one clear effect, which occurred on $\mathrm{d} 2$ of testing during the second 5 -min test interval $(p<0.01)$. During this interval, the PKU group was less active than either of the other groups, which did not differ from one another $(\overline{\mathrm{x}} \pm$ SEM, PKU $=118.5 \pm 6.3$; PKU-VIL $=139.8 \pm 5.0 ;$ PFC $=$ $134.3 \pm 4.5$; PKU $<$ PKU-VIL and PKU $<$ PFC by Duncan comparisons, both $p<0.05$ ).

No differences were found on the startle reflex using either auditory or tactile stimuli, with or without a prepulse response inhibition signal (Fig. 3).

\section{DISCUSSION}

The data revealed that the VIL treatment had a selective beneficial effect on CNS development in utero. The effect was noteworthy for its specificity and magnitude on learning ability. VIL had a large effect on the learning of a complex water maze, only a modest effect on locomotor activity, and no effect on

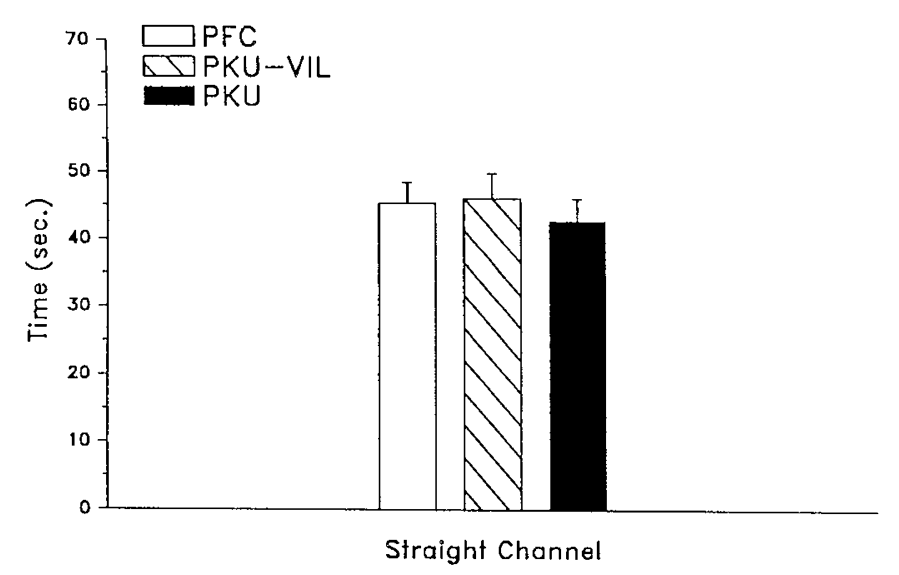

Fig. 1. Mean \pm SEM straight channel swimming times (s) summed across trials. There were no group differences found by ANOVA. Similarly, there were no differences when the data were analyzed separately by last trial or fastest trial. All litters were coded and tested by personnel blind to treatment group on this and all other tests. PKU = offspring of 10 dams given $2.5 \%$ L-phe $+0.12 \%$ PCPA in the diet on $\mathrm{d} 5-15$ of gestation; PKU-VIL = offspring of 12 dams given the PKU group's diet supplemented with a mixture of valine, isoleucine, and leucine ( $\mathrm{L}-$ isomers) on the same days of gestation; $\mathrm{PFC}=$ offspring of 12 control dams given standard diet in the amount consumed by the dams in the PKU group (pair-fed).

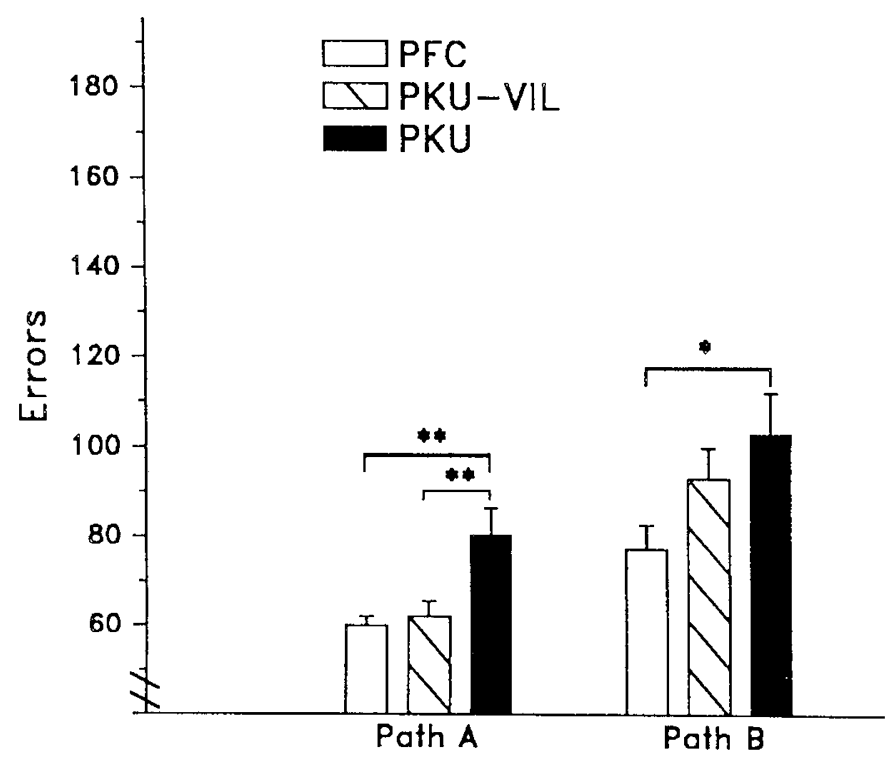

Fig. 2. Mean \pm SEM of the sum of cul-de-sac errors committed in the water maze during each phase of testing ( 6 trials/phase). Group differences were found on path $\mathrm{A}(p<0.001)$ and path $\mathrm{B}(p<0.05)$ by fixed-effect factorial ANOVA. Individual group comparisons were made using Duncan's multiple range test. Duncan comparisons are shown as ${ }^{*} p<0.05$ and ${ }^{* *} p<0.01$. An essentially identical pattern was found for excursion errors, i.e. group differences were found for path $\mathrm{A}(p<0.05)$ and path $\mathrm{B}(p<0.05)$. For excursion errors, group differences for path A were $(\overline{\mathrm{X}} \pm \mathrm{SEM}): \mathrm{PKU}=43.5 \pm 3.2, \mathrm{PKU}-\mathrm{VIL}=35.7 \pm 2.0, \mathrm{PFC}=$ $35.4 \pm 1.2$ (PKU $>$ PKU-VIL or PFC, both $p<0.05$ ); for path B: PKU $=70.7 \pm 5.5, \mathrm{PKU}-\mathrm{VIL}=62.8 \pm 3.7, \mathrm{PFC}=55.1 \pm 3.4(\mathrm{PKU}>\mathrm{PFC}$ $p<0.05$, PKU-VIL $<$ PKU, NS, and PKU-VIL $>$ PFC, NS, all by Duncan comparisons). The same pattern was also seen for maze times (not presented), but differences were smaller, i.e. the group F-ratio for path $\mathrm{A}$ was $p<0.06$ and for path $\mathrm{B}$ it was $p<0.10$. Individual groups were aligned in the same pattern on times as they were for both types of errors on each phase of this test.

swimming ability or the startle reflex. The protective effect of VIL on learning was most evident and only significant in terms of acquisition of the maze problem (path A). The basis of the beneficial effects of VIL on in utero development remains to be determined. There is preliminary data suggesting that VIL may reduce fetal brain phenylalanine content in PKU-VIL rats (18).

No phenylalanine-only or PCPA-only exposed groups, no phenylalanine-VIL or PCPA-VIL groups, no VIL-only or VIL ad libitum-fed groups, and no unsupplemented ad libitum-fed groups were included in the present investigation for several reasons. First, the inclusion of such controls in an investigation prior to determining the existence of a PKU-VIL effect would be counterproductive. Second, the purpose of this investigation was not to test the VIL supplement against phenylalanine-alone or PCPA-alone, but to assess its effects compared to a specific and established model of induced PKU that utilizes a particular combination of phenylalanine and PCPA dosages. Third, as both phenylalanine and PCPA have been shown not to produce an adequate biochemical model of PKU when used separately, and as neither have ever been shown to produce the types of behavioral effects assessed herein, there was little to be gained by the inclusion of these extensive additional control groups. Given that the data now support the potential of the VIL supplement in a model of maternal PKU, we will conduct further experiments, one of which will be to determine whether any plausible alternative combination or permutation of treatments can produce a comparable outcome.

As effective as the VIL supplement was at preventing the untoward effects of maternal hyperphenylalaninemia on maze 


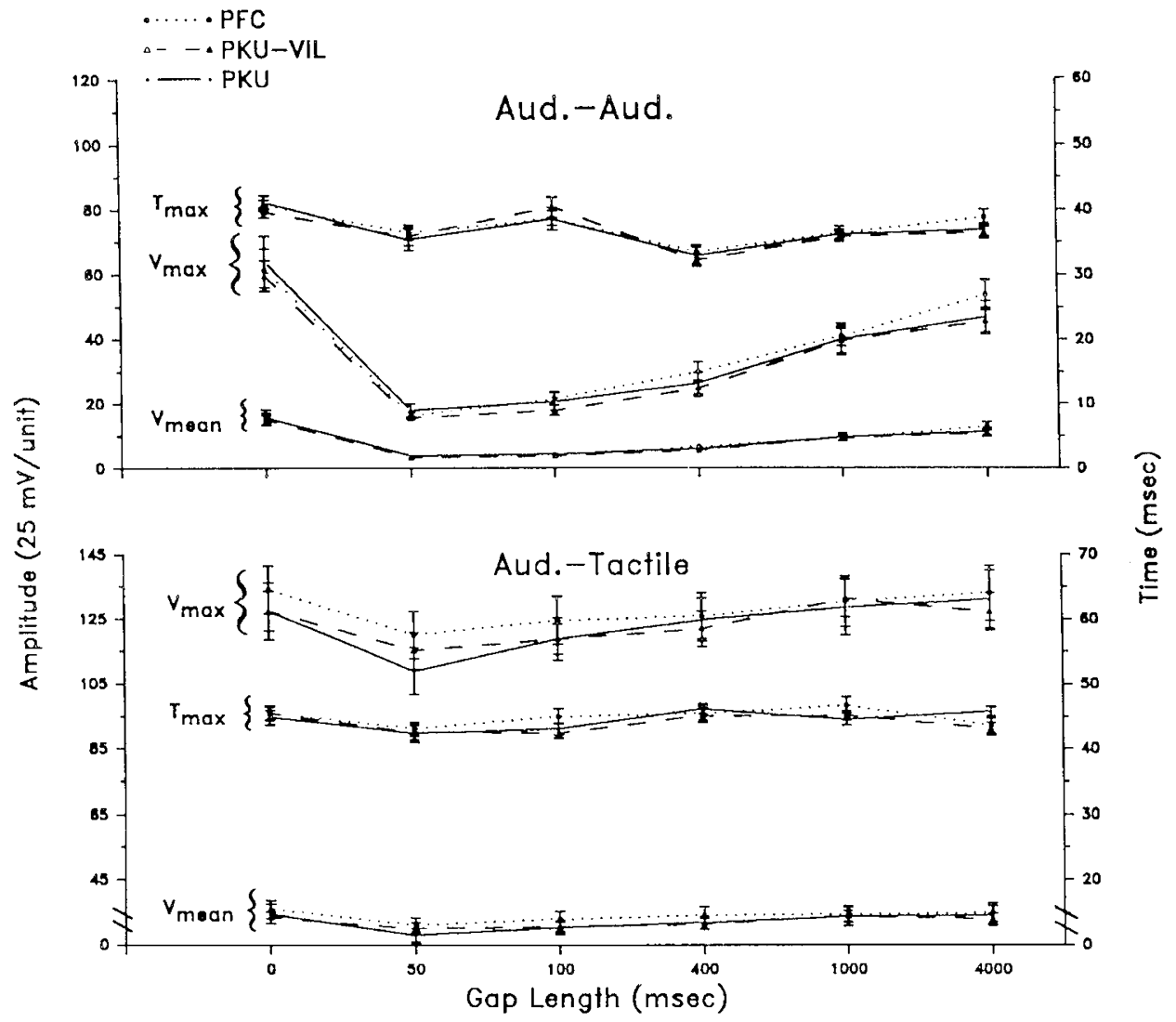

Fig. 3. Mean \pm SEM startle responses shown across various prepulse gap lengths (ms). Upper, responses to an auditory startle-eliciting stimulus presented following an auditory prepulse signal. Lower, responses to a tactile startle-eliciting stimulus presented following an auditory prepulse signal. Mixed model factorial ANOVA for each stimulus condition revealed no significant group or group-related interactions on any of the three dependent measures ( $\mathrm{V}_{\max }, \mathrm{V}_{\text {mean }}$, or $\mathrm{T}_{\max }$ ) recorded of the startle response.

acquisition, its effect on maze reversal learning was less clear. The PKU-VIL group did not commit significantly more errors on reversal than controls, suggesting a normalizing effect of the VIL supplementation. By contrast, the PKU-VIL group did not commit significantly fewer errors on reversal than the PKU group, suggesting no improvement. As the PKU and control groups differed from one another, it is not clear from these data whether the intermediate performance of the PKU-VIL group represents a partial effect of VIL or no effect. However, in a recently completed replication and extension of these data, a significant improvement in Olton radial-arm maze performance was found in the PKU-VIL group compared to the PKU group (Vorhees CV, Weisenberg KD, Acuff WP, and Berry HK, unpublished observations).

It remains to be seen whether a slightly different dose of the VIL mixture or a change in the proportions of the constituent branched-chain amino acids would be more effective than the present regimen. It also remains possible that combining VIL with other LNAA might enhance the efficacy of this general approach. An issue of equal importance is whether the cognitive improvements demonstrated here in the expanded Biel maze will generalize to other tests of cognitive performance, such as the Morris hidden-platform test of spatial relations learning.

The present data suggest a new approach for the treatment of maternal PKU. The data must be interpreted with caution, however, as a single test of rodent learning cannot assure that human cognitive development would be comparably protected, nor has the present research addressed the other sequelae of maternal PKU. Furthermore, the present data cover a period of rat development that would be comparable to only the first 8$12 \mathrm{wk}$ of human development, i.e. the period of major organogenesis. This may not cover all the stages of CNS development affected by maternal PKU, as maternal PKU results in chronic hyperphenylalaninemia, which extends from prior to conception to and beyond parturition. Maternal PKU also induces congenital heart defects, microcephaly, and intrauterine growth retardation, and research is underway on the effects of the current model on cardiogenesis and growth (19).

Acknowledgments. The authors express their appreciation to C. A. Blanton, M. S. Moran, and S. Champlin for assistance in the conduct of this research, and to Drs. R. L. Brunner, J. J. Hutton, E. Mollnow, and P. St.J. Dignan, for critical comments on previous versions of the manuscript.

\section{REFERENCES}

1. Tourian AY, Sidbury JB 1978 Phenylketonuria. In: Stanbury JB, Wyngaarden JB, Frederickson DS (eds) The Metabolic Basis of Inherited Diseases. McGraw-Hill, New York, pp 240-255

2. Lenke RR, Levy HL 1980 Maternal phenylketonuria and hyperphenylalaninemia: an international survey of the outcome of untreated and treated pregnancies. N Engl J Med 303:1202-1208

3. Levy HL, Lenke RR, Crocker AC (eds) 1981 Maternal PKU: Proceedings of a Conference. US Department of Health and Human Services, Rockville, MD pp $1-90$

4. Levy HL, Waisbren SE 1983 Effects of untreated maternal phenylketonuria and hyperphenylalaninemia on the fetus. N Engl J Med 309:1269-1274

5. Huntley CC, Stevenson RE 1969 Maternal phenylketonuria: course of two pregnancies. Obstet Gynecol 34:694-700

6. Lipson A, Beuhler B, Bartley J, Walsh D, Yu J, O'Halloran M, Webster, W 1984 Maternal hyperphenylalaninemia fetal effects. J Pediatr 104:216-220

7. Murphy D, Saul I, Kirby M 1985 Maternal phenylketonuria and phenylalanine restricted diet: studies of 7 pregnancies and of offsprings produced. Ir J Med Sci 154:66-70

8. Oldendorf WH 1973 Saturation of blood brain barrier transport of amino acids in phenylketonuria. Arch Neurol 28:45-48

9. Pardridge WM, Oldendorf WH 1975 Kinetic analysis of blood-brain barrier transport of amino acids. Biochim Biophys Acta 401:128-136 
10. Fernstrom JD 1983 Role of precursor availability in control of monoamine biosynthesis in brain. Physiol Rev 63:484-546

11. Pardridge WM 1983 Brain metabolism: a perspective from the blood-brain barrier. Physiol Rev 63:1481-1535

12. Smith QR 1987 Kinetic analysis of neutral amino acid transport across the blood-brain barrier. In: Kaufman, $\mathrm{S}$ (ed) Amino Acids in Health and Disease Alan R. Liss, New York, pp 65-85

13. Airaksinen MM, Leppanen ML, Turakka H, Marvola M, MacDonald EJ 1975 Protective effect of tryptophan on experimental phenylketonuria induced with phenylalanine $+p$-chlorophenylalanine in rats. Med Biol 53:481-488

14. Andersen AE, Avins L 1976 Lowering brain phenylalanine levels by giving other large neutral amino acids: a new experimental therapeutic approach to phenylketonuria. Arch Neurol 33:684-686

15. Hughes JV, Johnson TC 1977 Hyperphenylalaninemia: effects on brain polyribosomes can be partially reversed by other amino acids. Science 195:402404

16. Kaufman S 1977 Phenylketonuria: biochemical mechanisms. Adv Neurochem 2:1-132

17. Lou H 1985 Large doses of tryptophan and tyrosine as potential therapeutic alternative to dietary phenylalanine restriction in phenylketonuria. Lancet 2:150-151

18. Berry HK, Butcher RE, Brunner RL, Bray NW, Hunt MM, Wharton CH 1977 New approaches to treatment of phenylketonuria. In: Mittler P (ed) Research to Practice in Mental Retardation, Biomedical Aspects, vol 3. University Park Press, Baltimore, pp 229-239

19. Brunner RL, Vorhees CV, McLean MS, Butcher RE, Berry HK 1978 Beneficial effect of isoleucine on fetal brain development in induced phenylketonuria. Brain Res 154:191-195

20. McSwigan JD, Vorhees CV, Brunner RL, Butcher RE, Berry HK 1981 Amelioration of maze deficits from induced hyperphenylalaninemia in adult rats using valine, isoleucine, and leucine. Behav Neural Biol 33:378-384

21. Berry HK, Bofinger MK, Hunt MM, Phillips PJ, Guilfoile MB 1982 Reduction of cerebrospinal fluid phenylalanine after oral administration of valine, isoleucine, and leucine. Pediatr Res 16:751-755

22. Jordan MK, Brunner RL, Hunt MM, Berry HK 1985 Preliminary support for the oral administration of valine, isoleucine and leucine for phenylketonuria. Dev Med Child Neurol 27:33-39

23. Butcher RE 1970 Learning impairment associated with maternal phenylketonuria in rats. Nature 226:555-556

24. Butcher R, Vorhees C, Berry H 1970 A learning impairment associated with induced phenylketonuria. Life Sci 9:1261-1268

25. Berry HK, Butcher RE, Kazmaier KJ, Poncet IB 1975 Biochemical effects of induced phenylketonuria in rats. Biol Neonate 26:88-101

26. Koe BK, Weissman A 1966 p-Chlorophenylalanine: a specific depletor of brain serotonin. J Pharmacol Exp Ther 154:499-516

27. Lipton MA, Gordon R, Guroff G, Udenfriend S 1967 p-Chlorophenylalanineinduced chemical manifestation of phenylketonuria in rats. Science 156:248250

28. Butcher RE 1969 Behavioral effects of maternal phenylketonuria in rats. Doctoral Dissertation, University of Cincinnati, pp I-49

29. Andersen A 1976 Maternal hyperphenylalaninemia: an experimental model in rats. Dev Psychobiol 9:157-166

30. Vorhees CV, Butcher RE, Berry HK 1981 Progress in experimental phenylketonuria: a critical review. Neurosci Biobehav Rev 5:177-190

31. Buelke-Sam J, Kimmel CA, Adams J, Nelson CJ, Vorhees CV, Wright DC, St. Omer V, Karol BA, Butcher RE, Geyer MA, Holson JF, Kutscher CL, Wayner MJ 1985 Collaborative Behavioral Teratology Study: results. Neurobehav Toxicol Teratol 7:591-624

32. Vorhees CV 1987 Maze learning in rats: a comparison of performance in two water mazes in progeny prenatally exposed to different doses of phenytoin. Neurotoxicol Teratol 9:235-241

33. Benson JV, Gordan MJ, Patterson JA 1967 Accelerated chromatographic analysis of amino acids in physiological fluids containing glutamine and asparagine. Anal Biochem 18:228-240

34. Berry HK 1976 Hyperphenylalaninemias and tyrosinemias. Clin Perinatol $3: 15-40$ 